\title{
Technical aspects and early results of robotic esophagectomy with chest anastomosis
}

\author{
Robert James Cerfolio, MD, FACS, FCCP, ${ }^{\mathrm{a}, \mathrm{b}}$ Ayesha S. Bryant, MSPH, MD, ${ }^{\mathrm{b}}$ and \\ Mary T. Hawn, MD, MPH, FACS
}

Objectives: Minimally invasive esophagectomy with a chest anastomosis has advantages. We present technical lessons learned and early results.

\begin{abstract}
Methods: A retrospective review was conducted of minimally invasive laparoscopic and robotic Ivor Lewis esophagectomy.

Results: Over 10 months, 22 patients (19 men) underwent laparoscopic gastric mobilization, with robotic esophagectomy. All had the thoracic portion completed robotically and 21 had the stomach mobilized laproscopically. All had esophageal cancer and 20 received neoadjuvant chemoradiotherapy. All had R0 resection with a median of 18 lymph nodes removed and a blood loss of $40 \mathrm{~mL}$. The first 6 patients underwent a stapled posterior and hand-sewn anterior anastomosis; five of these patients experienced a major morbidity, including 1 anastomotic leak and 1 leak from the gastric staple line. The last 16 patients had a 2-layered completely hand-sewn anastomosis, and there were no anastomotic leaks or major morbidities. There were no 30- or 90-day mortalities. Technical improvements included placing a loop around the esophagus in the abdomen for third arm retraction, advancing the gastric conduit into the chest using nonrobotic instruments, using 10-cm nonabsorbable interrupted sutures for the outer layer, and a running $22-\mathrm{cm}$ long absorbable suture for the inner layer.
\end{abstract}

Conclusions: Robotic thoracic esophagectomy using ports only is feasible, safe, and affords R0 resection with thorough thoracic lymph node dissection. It also allows the sewing of a 2-layered chest anastomosis with good early results. (J Thorac Cardiovasc Surg 2013;145:90-6)

Minimally invasive esophagectomy (MIE) is attractive and may offer advantages compared with resections via open techniques. ${ }^{1,2}$ However, there remains considerable debate about the optimal location and manner to perform the anastomosis (eg, neck vs chest and stapled vs handsewn) ${ }^{3,4}$ Recently, Luketich, one of the pioneers of MIE who has performed over 500 MIEs with an anastomosis in the neck, has switched his technique to a chest anastomosis between the esophagus and the gastric conduit. ${ }^{5,6}$ We have always preferred a chest anastomosis because we believe there is less recurrent laryngeal nerve injury, fewer aspirations, and a lower leak rate. However, the ideal approach is unknown and is often patient dependent. ${ }^{7}$ In addition to the location of the anastomosis,

From the Divisions of Thoracic Surgery, ${ }^{\mathrm{a}}$ Cardiothoracic Surgery, ${ }^{\mathrm{b}}$ and Gastrointestinal Surgery, ${ }^{\mathrm{c}}$ Department of Surgery, University of Alabama at Birmingham, Birmingham, Ala.

Disclosures: Robert J. Cerfolio has received money to speak for Intuitive Surgical (Sunnyvale, Calif), proctor surgeons for robotic surgery, and has taught robotic training sessions. The other authors have nothing to disclose with regard to commercial support.

Received for publication Dec 31, 2011; revisions received March 9, 2012; accepted for publication April 4, 2012; available ahead of print Aug 20, 2012.

Address for reprints: Robert James Cerfolio, MD, FACS, FCCP, Division of Cardiothoracic Surgery, UAB, 703 19th St S, ZRB 739, Birmingham, AL 35294 (E-mail: rcerfolio@uab.edu).

0022-5223/\$36.00

Copyright (c) 2013 by The American Association for Thoracic Surgery http://dx.doi.org/10.1016/j.jtcvs.2012.04.022 the best manner in which to construct the anastomosis is also highly controversial. Some advocate a completely stapled technique using a circular stapling device; others suggest a partially stapled anastomosis posteriorly that is hand-sewn anteriorly, and others favor a completely handsewn 2-layered anastomosis. $\mathrm{We}^{8}$ have shown an extremely low anastomotic leak rate with our open Ivor Lewis esophagogastrectomy, 2-layered hand-sewn technique, with only $1(0.4 \%)$ leak among 221 patients. However, these anastomoses were performed via thoracotomy and laparotomy, and unfortunately many patients had other serious complications with high mortality, possibly attributable to the surgical approach as reported in our paper. ${ }^{8}$ Thus our preference for the anastomosis has remained in the chest and hand-sewn, but this is difficult to perform using video-assisted thoracoscopic surgery (VATS). The purpose of this study is to communicate the technical aspects, lessons learned, and early results of our completely portal, robotic Ivor Lewis esophagectomy (CPR-ILE) using a totally hand-sewn 2-layered anastomosis.

\section{METHODS}

This is a retrospective study using a prospective database of a consecutive series of patients who were scheduled for (intent to treat) a completely portal thoracic robotic operation for esophageal cancer. The entry criteria for this study were as follows: age greater than 19 years old and a preoperative plan to undergo a completely portal robotic esophagectomy with a chest anastomosis. Patients who had a previous right thoracotomy (since 


\section{Abbreviations and Acronyms \\ CPR- = completely portal, robotic Ivor Lewis \\ ILE esophagectomy \\ CPR- = completely portal, robotic Ivor Lewis \\ ILE-4 esophagectomy using 4 arms \\ $\mathrm{CT}=$ computed tomography \\ EUS = esophageal ultrasound \\ MIE = minimally invasive esophagectomy \\ PET = positron emission tomograpy \\ VATS $=$ video-assisted thoracoscopic surgery}

a robotic approach was not chosen) and those who had cancerous lesions that were proximal to $23 \mathrm{~cm}$ from the incisors were not eligible for CPRILE. These latter patients underwent robotic mobilization of the esophagus first and then a transhiatal operation with an anastomosis constructed in the left side of the neck. However, patients with the intent to have a robotic chest operation or intent to have a laparoscopic abdominal operation were included.

\section{Nomenclature}

An international robotic committee has been formed. It met, developed an agreed on nomenclature, and submitted an article that describes robotic thoracic operations.* These agreed on terms and definitions are used throughout this article. The nomenclature describes the technique, the operation, and the number of robotic arms used. It is currently applicable to the da Vinci robotic system (Intuitive Surgical, Sunnyvale, Calif) used in this study, but the nomenclature is flexible and was written to be applicable for any new robotic system. The operation used in this study has been designated a completely portal, robotic Ivor Lewis esophagectomy (CPR-ILE) using $4 \mathrm{arms}^{4}$ or "CPR-ILE-4" for short. Completely portal is defined as having the entire operation (except for specimen removal) performed via trocars or ports only (no access incisions) and the lack of communication of the ambient air in the operating room with the pleural space. The nomenclature does not take into account the abdominal aspect of the operation, which in this series was performed using laproscopic techniques initially for all patients.

All patient information was entered in our prospective database. The details of this patient database and the methods used for obtaining follow-up data have been previously reported elsewhere. ${ }^{9}$ In brief, the database is entered on the day of surgery except for the details of the postoperative course. It contains all variables collected by The Society of Thoracic Surgeons database and adheres to the same rigors of verification and validation of data accuracy and entry. In addition, other aspects of patient information are collected, some of which include 90-day mortality, genetic and molecular markers of tumors resected, and long-term follow-up information.

Patients with biopsy-proven esophageal cancer had a chest computed tomography (CT) scan within 40 days of surgery, an integrated positron emission tomography/computed tomography (PET-CT), and an esophageal ultrasound (EUS) before the induction of neoadjuvant therapy. In general, neoadjuvant chemoradiotherapy was used for patients with T2 or greater or $\mathrm{N} 1$ or greater disease (exception being patients $>75$ years of age with $\mathrm{T} 2$ NO M0 lesions). All patients had a restaging, repeat integrated PET-CT and EUS after the completion of their neoadjuvant therapy. Repeat EUS was used to biopsy and/or to prove recalcitrant celiac axis nodal disease Patients were selected for surgery on the basis of their oncologic stage and their fitness for the operation as previously described by us. ${ }^{8}$

* Cerfolio RJ, Dylewski M, Parks, B. International consensus paper for nomenclature for robotic pulmonary resection. Submitted for publication.
The reasons to convert from a completely portal robotic operation to an open thoracotomy were as follows: bleeding that could not be controlled robotically, the inability to enter the pleural space and insufflate carbon dioxide secondary to pleural symphysis from adhesions, or the inability to completely remove the tumor and achieve an $\mathrm{R} 0$ resection. The reason to convert from a laparoscopic approach to a laparotomy was the inability to tubularize the stomach with the stapler.

The University of Alabama at Birmingham's Institutional Review Board approved this protocol as well as the prospective database used to collect information for this study (X030403013). Individual patient consent was waived for inclusion in this study; however, patient consent was obtained to enter patient information into the prospective database.

\section{Operative Technique}

The stomach is mobilized via a laparoscopic, robotic, or open approach. In this series the laparoscopic approach only was used and was performed by a team of gastrointestinal surgeons led by M.T.H.

With the patient in the supine, reverse Trendelenburg position, four $5-\mathrm{mm}$ and one 12- $\mathrm{mm}$ trocars are placed in the upper abdomen. The 5 -mm trocar to the left of the umbilicus is then used for the feeding jejunostomy at the completion of the gastric mobilization. The stomach's blood supply is based on the right gastroepiploic arterial arcade. The conduct of the abdominal aspect of our Ivor Lewis procedure has been previously chronicled. ${ }^{8}$ We do not typically perform a Kocher maneuver unless a possible neck anastomosis is needed. The pylorus is injected with botulinum as previously described and a feeding jejunostomy tube is routinely placed. ${ }^{8}$ Abdominal lymph nodes are removed from around the left gastric artery and vein and swept toward the resected specimen. In addition, the lesser omentum is resected as well. The stomach is tubularized in the abdomen by stapling it from the lesser curve moving cephalad toward the fundus. The gastroesophageal junction is resected and the margin is checked to ensure it is negative. The divided stomach is sewn to the end of the divided esophagus and a soft wide drain is sutured around the esophagus in the abdomen. The hiatus is opened slightly toward the right side of the chest and the conduit and drain are placed into the lower aspect of the right side of the chest.

Once the abdominal aspect of the operation is completed, the patient is placed in the left lateral decubitus position with the right side of the chest up. The patient is titled forward to allow the lung and blood to fall away from the posterior mediastinum. Prone positioning is not used because of the added anesthesia and patient positioning time. The robotic ports are placed as shown in Figure 1. The first port inserted is a camera port that is placed $9 \mathrm{~cm}$ inferiorly to the robotic arm 1, which is just below the hair line of the right axilla. The camera is placed first to ensure that there are no adhesions and that the pleural space is free. Humidified warm carbon dioxide is insufflated (Insuflow, Lexicon Medical, St Paul, Minn) at a pressure of 8 to $10 \mathrm{~mm} \mathrm{Hg}$. This lowers the diaphragm, yields a larger working space, and helps prevent desiccation of tissue. In general, all of the remaining incisions are not made until all 4 planned robotic ports have been marked out with a pen on the skin, carefully measured and a seeking needle placed in each one, and local anesthetic injected. We also perform a formal paraveterbral block by injecting multiple intercostal nerves posteriorly using a 21 -gauge needle filled with $0.25 \%$ bupivacaine with epinephrine before making any other incisions except for the camera port. The second port placed is an 8-mm port for the robotic arm 1. This port will serve as the surgeon's right hand. The instruments commonly implemented in this arm are the robotic cautery and the Maryland bipolar. It is $9 \mathrm{~cm}$ superior to the camera port in the anterior axillary line but slightly more anterior, as shown in Figure 1. This incision should be made just inferior to the right axillary hair line (not in the axilla but just inferior to it) and just medial to the anterior edge of the scapula. The third incision made is also an 8-mm port for robotic arm 2. This arm acts as the surgeon's left hand. The instrument used in this arm is almost exclusively the Cadiere forceps (Intuitive Surgical). It too is in the anterior axillary line and it is $9 \mathrm{~cm}$ inferior to the camera 

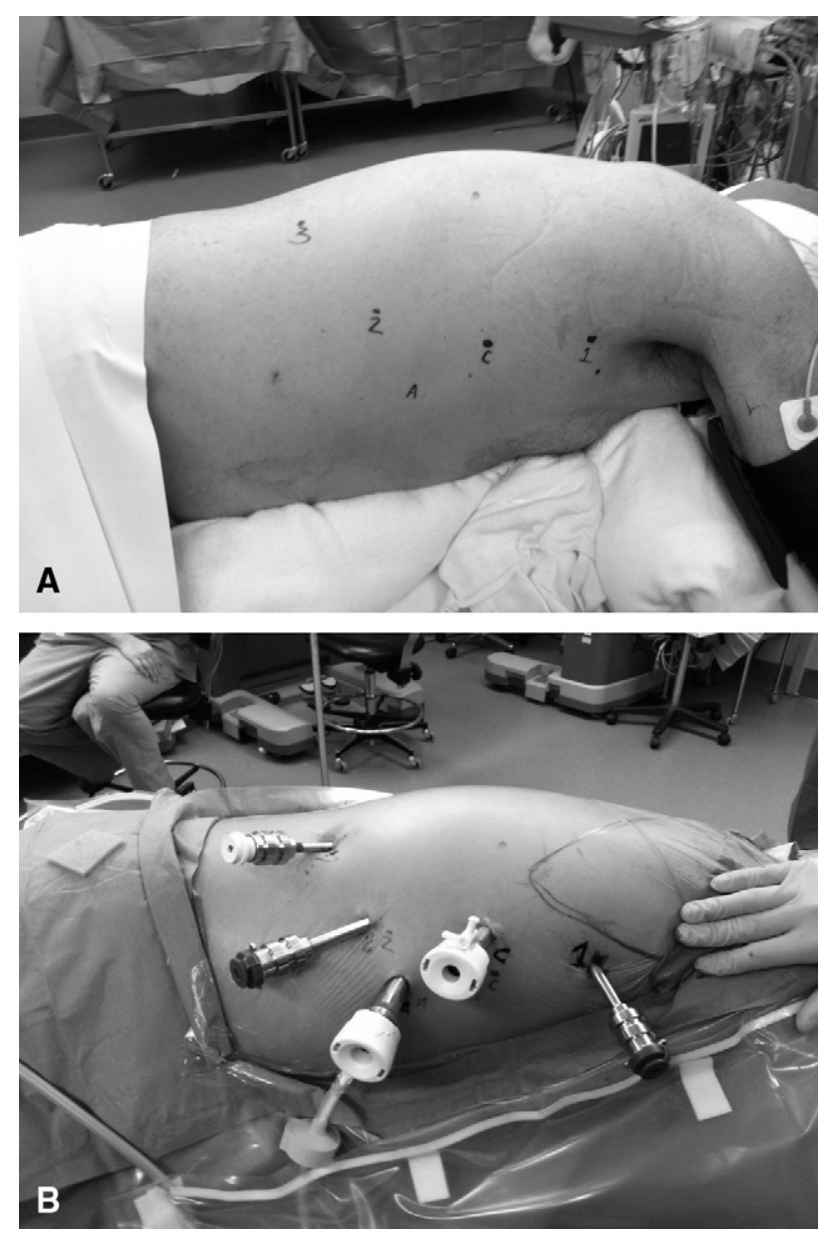

FIGURE 1. A and B, Port placement for the robotic thoracic part of a completely portal robotic (CPR) Ivor Lewis esophagectomy (ILE) using 4 arms, CPR-ILE-4. $C$ is for the camera port and $A$ is for the nonrobotic access port site.

port. However, it should be positioned slightly posterior to the ports of robotic arm 1 and the camera port to allow amble room for the nonrobotic port that is used by the bedside assistant. This port is a 12 - or $15-\mathrm{mm}$ trocar that is used to suck blood from the field and hand the surgeon sutures and sponges. It is later enlarged to remove the specimen. The third port placed is the smaller 5-mm port. It is the most posterior port and is for robotic arm 3 . It is $10-\mathrm{cm}$ away from robotic arm 2 and more posterior, as shown in Figure 1 . We have only placed a 5 -mm lung grasper in this port.

The robot is driven over the patient's back on a slight $30^{\circ}$ angle. This allows for visualization of the entire thoracic esophagus and posterior mediastinum of the chest from the diaphragm to the apex of the chest. The ports are docked to the robot and the resection is started. The esophagus is taken off the aorta by using the third arm. It retracts the esophagus anteriorly and cephalad via a drain placed around it, and the arterial branches are ligated with the bipolar cautery or clips. The azygos vein is divided via a vascular stapler that is placed via the nonrobotic port. The esophagus is divided well above the divided azygos vein stump, and the tumor, esophagus, and proximal stomach are extracted after placing the specimen in an Anchor bag (Progressive Medical, Addison, Ill). After bagging, the specimen it is removed via the 1 nonrobotic port, which often has to be enlarged. After specimen removal, carbon dioxide insufflation is reestablished by placing a small Steri-Drape surgical drape (3M Company, St Paul, Minn) over the enlarged incision and the port is placed through it or by placing a few sutures around the skin to keep carbon dioxide from escaping. The proximal esophageal margin is sent to the pathology department for frozen section to ensure that it is free of cancer and Barrett cells.

\section{Hand-Sewn 2-Layered Robotic Chest Anastomosis}

The tubularized stomach is then carefully brought into the chest with a Scanlan clamp (Scanlan International, St Paul, Minn) that is introduced from the nonrobotic port. The conduit is positioned above the divided azygos vein and under the divided esophagus. A location on the tubularized gastric conduit, at least 3- to 4-cm below the most superior aspect of the gastric staple line and as far away as possible from the lesser curve staple, is selected for the gastrotomy for the anastomosis. This area must be carefully selected. It should avoid tension on the anastomosis and it should minimize arterial ischemia by maximizing the distance between the staple lines and the gastrotomy. The back layer of the posterior aspect of the anastomosis is constructed first using 10-cm long, interrupted 3-0 silk sutures (Ethicon, Inc, Somerville, NJ), as shown in Figure 2, A. The robotic instruments used during the anastomosis are a robotic fine-tipped needle driver in robotic arm 1, which serves as the right hand, and a robotic long-tip needle forceps in robotic arm 2, which serves as the left hand. This instrument is now used because it is less traumatic than a needle driver in the left hand, which is important when using a running suture (as is used in the inner layer). Usually 5 interrupted sutures are placed in the back row and sew the postmuscular layer of the esophagus to the serosal layer of the stomach.

The inner layer of the anastomosis is constructed next by using a running layer of 22-cm long 3-0 polydiaxone sutures (PDS, Ethicon, Inc). We prefer starting on the medial part of the anastomosis at the 3 o'clock location. This knot is placed extraluminally and then the suture is run moving away from the surgeon from the 3 o'clock to the 9 o'clock location as shown in Figure 2, $B$. Once the posterior inner layer of the anastomosis is completed, a second PDS suture that is also $22-\mathrm{cm}$ long is started. It runs from the posterolateral aspect of the inner part of the anastomosis at the 9 o'clock location back toward the surgeon anteriorly. The nasogastric tube is inserted under direct vision before finishing this anterior inner layer. Finally, the second layer of the anterior part of the anastomosis is completed by placing several interrupted 3-0 silk sutures anteriorly, as shown in Figure 2, C. We then buttress the anterior aspect of the anastomosis by sewing some of the excess omentum that is intentionally left on the greater curve (gastroepiploic side) of the stomach.

Morbidity was defined using the definitions from The Society of Thoracic Surgeons database. Operative mortality was defined as death within 30 days after surgery from any cause or before discharge. The 7th edition of the TNM staging classification was used. Data were stored in Excel (Microsoft Corp, Seattle, Wash) and descriptive statistics are shown by the use of medians, standard deviations, and frequencies as appropriate.

\section{RESULTS}

Between April 2011 and February 2012, a total of 28 patients were scheduled to undergo esophagectomy. Of these, 2 patients underwent transhiatal esophagectomy, 2 had a planned right thoracotomy, and 2 had metastatic disease found in the abdomen. The remaining 22 patients met the entry criteria for this study and were scheduled to undergo a CPR-ILE-4 using a laparoscopic abdominal approach, robotic chest approach, and a chest anastomosis. The patient characteristics are shown in Table 1. The postoperative outcomes are shown in Table 2 and are stratified by method of anastomosis (hand-sewn compared with stapled). There was a greater rate of postoperative morbidities in the stapled group as compared with the hand-sewn group. Three patients experienced a minor morbidity: one patient had 

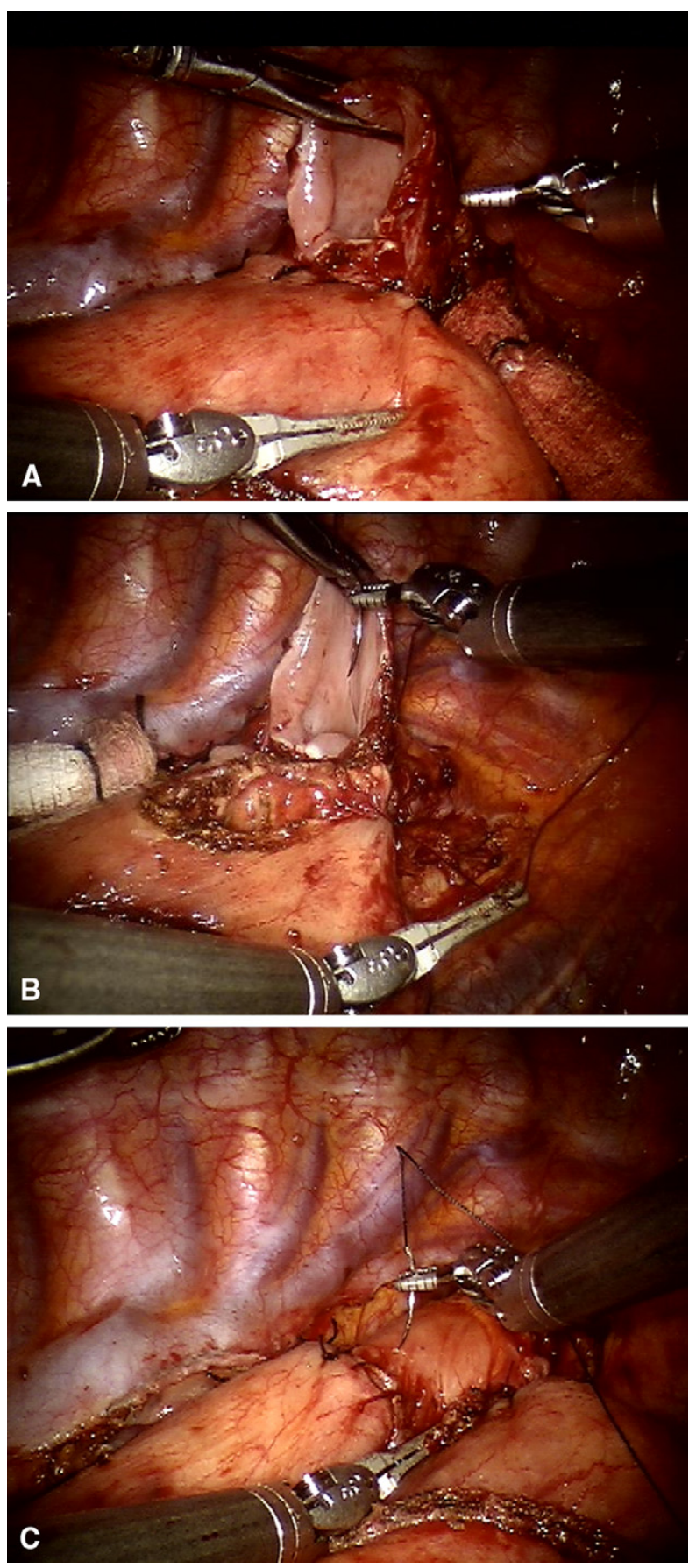

FIGURE 2. A, Intraoperative photograph shows the esophagus being held open by a 5-mm lung grasper that is placed through the most posterior 5 $\mathrm{mm}$, via robotic arm 3 . The back row of interrupted sutures is completed. $\mathrm{B}$, Intraoperative photograph depicts the inner layer of the anastomosis being performed using a running 3-0 absorbable suture (knots are outside the lumen) and sewing mucosa to mucosa. Note how robotic arm 2, which has a nontraumatic long-tipped needle forceps, enables the surgeon to maintain tension on the running suture without fraying as the needle driver (in robotic arm 1) is able to place the sutures. C, Intraoperative photo that depicts the second anterior layer of interrupted sutures being placed, which sews the muscular layer of the esophagus to serosa of the gastric conduit using interrupted nonabsorbable sutures. transient atrial fibrillation, another had urinary retention in the stapled group, and a third patient in the hand-sewn group also had transient atrial fibrillation. Complete (R0) resection was accomplished in all patients. No patients required blood transfusion. The median number of lymph nodes removed was 18 (range, 15-26). There were no conversions from a robotic chest approach to thoracotomy; however, there was 1 conversion from a laparoscopy to a laparotomy. This occurred in a patient with extensive thickening of the stomach wall resulting in breakdown of the staple line. We performed a laparotomy and finished resecting the upper stomach and lower esophagus and oversewed the entire staple line. The margin was negative for cancer. The thick tissue was thought to be a result of the radiation and not cancer inasmuch as the tumor was $30 \mathrm{~cm}$ from the incision and, although it a T3 N1 lesion, initially it was only $3 \mathrm{~cm}$ long.

The operative times for the 16 patients who underwent a hand-sewn anastomosis are shown in Figure 3. It shows the times for the laparoscopic part, the robotic chest part, and the hand-sewn anastomosis. The median operative time (defined as time from skin incision until skin closure) was 6 hours 7 minutes (range, 4 hours 50 minutes to 7 hours 33 minutes). The median times for the total operation and chest portion of the operation decreased progressively, as shown in Figure 3. There were no 30- or 90-day operative mortalities in this series. Follow-up was complete in all patients; none has had a local or systemic recurrence although the median follow-up is only 5 months.

\section{DISCUSSION}

Patients are increasingly seeking minimally invasive surgery over open techniques because of reduced morbidity. However, the best way to perform an MIE remains unknown. The surgical approach chosen is often based on surgeon preference instead of patient anatomy or characteristics. However, for adenocarcinomas of the lower gastroesophageal junction, the surgeon's preference (neck vs chest anastomosis) is reasonable. Some surgeons favor a robotic or VATS mobilization of the esophagus and then, after patient repositioning, perform a laparoscopic mobilization of the stomach followed by an esophagogastric anastomosis in the left side of the neck. Others prefer to remain out of the neck and favor a chest anastomosis. Few surgeons perform a minimally invasive Ivor Lewis procedure with a chest anastomosis. Most important, the surgeon and his or her team need to be able to perform all of these and other types of esophagectomy well because the location of the patient's tumor and other factors often dictate the best surgical approach for each patient.

The best way to perform the esophagogastric anastomosis is also often debated. Many favor stapling the back part of the anastomosis and hand-sewing the anterior aspect. This has several advantages. It decreases operative times and reduces the stricture rate. Orringer, Marshall, and 
TABLE 1. Patient characteristics of the 22 patients who underwent a robotic Ivor Lewis esophagectomy

\begin{tabular}{lc}
\hline Age (median, y) (range) & $66(47-77)$ \\
Gender & \\
Male & $19(86 \%)$ \\
Female & $3(14 \%)$ \\
Height (in), median & 69 \\
Weight (lb), median & 176 \\
Neoadjuvant chemoradiation therapy & $20(91 \%)$ \\
Weight loss in 3 mo before surgery (median lb) & $15(0-40)$ \\
Comorbidities & \\
Current smoker (quit $<1$ mo preoperatively) & $2(9 \%)$ \\
Pulmonary function test (median) & \\
FEV ${ }_{1} \%$ & $95 \%$ \\
DLCO (\%) & $87 \%$ \\
Hemoglobin, preoperative (g/dL) & 13.0 \\
Hypertension & $11(50 \%)$ \\
Heart disease and/or heart failure & $7(32 \%)$ \\
Prior cardiothoracic surgery & $3(14 \%)$ \\
Cerebral vascular accident/stroke & $2(9 \%)$ \\
Diabetes & $3(14 \%)$ \\
ASA class & $3(2-3)$ \\
ECOG (median) & $1(0-3)$ \\
Type of anastomosis & \\
Stapled in back & $6(27 \%)$ \\
Hand sewn & $16(73 \%)$ \\
\hline$F E V_{1} \%$, Forced expiratory volume in 1 second; $D L C O \%$, diffusion of carbon mon- \\
oxide; $A S A$, American Society of Anesthesiologists classification; $E C O G$, Eastern \\
Cancer Oncology Group. & \\
&
\end{tabular}

Iannettoni ${ }^{10}$ in 2000 reported a leak rate of only $3(2.7 \%)$ in 111 patients using this technique. Gorenstein, Bessler, and Sonnett ${ }^{11}$ in 2011 reported a leak rate of only $1(3.2 \%)$ in 31 patients. They used a completely stapled (not using an EEA stapler [Ethicon Endo-Surgery, Inc, Cincinnati, Ohio] but rather a linear stapling device) technique and showed excellent outcomes. These 2 techniques are fast and avoid having to hand-sew 2 layers. Hand-sewing increases operative time but may lead to a more precise and controlled gastrotomy. Thus there are many ways to perform the anastomosis and the key is experience and attention to detail.

In both our robotic and open esophagectomy experience, we have come to favor a completely hand-sewn 2-layered technique in the chest for patients with low esophageal cancer. This type of anastomosis may decrease conduit ischemia by allowing a more precise and controlled gastrotomy and avoid staple lines that may harbor low blood supply areas of the stomach. We prefer the chest because this approach lessens the injury to the left recurrent laryngeal nerve, lowers the leak rate, and functionally is not much different inasmuch as a high chest anastomosis is often only 2 to $3 \mathrm{~cm}$ below a left neck anastomosis.

The first description of a robotic esophagectomy was from Kernstine and colleagues ${ }^{12}$ in 2004. It was a case report on 1 patient. Bodner and coworkers ${ }^{13}$ in 2005 reported
TABLE 2. Postoperative outcomes by type of anastomosis: The first 6 patients had a stapled anastomosis and the last 16 patients had a handsewn anastomosis

\begin{tabular}{|c|c|c|}
\hline & $\begin{array}{c}\text { Stapled } \\
\text { anastomosis } \\
(\mathbf{n}=\mathbf{6})\end{array}$ & $\begin{array}{c}\text { Hand-sewn } \\
\text { anastomosis } \\
(\mathrm{n}=16)\end{array}$ \\
\hline \multicolumn{3}{|l|}{ Intraoperative characteristics } \\
\hline $\begin{array}{l}\text { Estimated blood loss, median } \\
\text { (range) }\end{array}$ & $75(40-800)$ & $60(30-120)$ \\
\hline $\begin{array}{l}\text { Lymph nodes removed, } \\
\text { median (range) }\end{array}$ & $17(15-26)$ & $18(15-28)$ \\
\hline $\begin{array}{l}\text { Conversion from robotic chest } \\
\text { to open operation approach }\end{array}$ & 0 & 0 \\
\hline \multicolumn{3}{|l|}{ Postoperative } \\
\hline $\begin{array}{l}\text { Hospital length of stay } \\
\text { (median, } d)\end{array}$ & $7(6-32)$ & $7(6-10)$ \\
\hline Major morbidity & $5(83 \%)$ & $0(0 \%)$ \\
\hline Leak, anastomotic & 1 & \\
\hline Leak, gastric & 1 & \\
\hline Empyema & 1 & \\
\hline Colon herniation & 1 & \\
\hline Chylothorax & 1 & \\
\hline Minor morbidity & $2(33 \%)$ & $1(6.3 \%)$ \\
\hline Atrial fibrillation & 1 & 1 \\
\hline Urinary retention & 1 & 0 \\
\hline $\begin{array}{l}\text { Reoperation during same } \\
\text { hospital stay }\end{array}$ & 5 & 0 \\
\hline \multicolumn{3}{|l|}{ Final pathology } \\
\hline \multicolumn{3}{|l|}{ Stage } \\
\hline Complete responder & $4(66 \%)$ & $9(56 \%)$ \\
\hline T1-4 N0 M0 & $1(17 \%)$ & $4(25 \%)$ \\
\hline T1-4 N1 (node) positive M0 & $1(17 \%)$ & $3(19 \%)$ \\
\hline \multicolumn{3}{|l|}{ Histology } \\
\hline Adenocarcinoma & $5(83 \%)$ & $13(81 \%)$ \\
\hline Squamous cell carcinoma & $1(17 \%)$ & $3(19 \%)$ \\
\hline
\end{tabular}

their experience using a robot-assisted thoracoscopic technique for esophagectomy cancer on 4 patients. Boone ${ }^{14}$ in 2009 presented a series of 47 patients who underwent robotic esophagectomy. All 3 groups started the operation in the right side of the chest robotically, mobilized the entire thoracic esophagus, and performed a right thoracic lymphadenectomy. They then placed their patients in a supine position and, after creating a gastric tube, performed an anastomosis in the left side of the neck. Their results were good. Puntambekar and associates ${ }^{15}$ in 2011 reported on 32 patients who underwent robotic esophagectomy. They too performed a neck anastomosis and reported excellent results. Finally, Weksler and coworkers ${ }^{16}$ in 2011 reported on 43 patients who underwent a robot-assisted MIE and reported comparable results to thoracic. These pioneers showed the feasibility and safety of robotic esophagectomy. However, all of these reports featured a neck anastomosis but few have shown how to do the anastomosis in the chest robotically. 


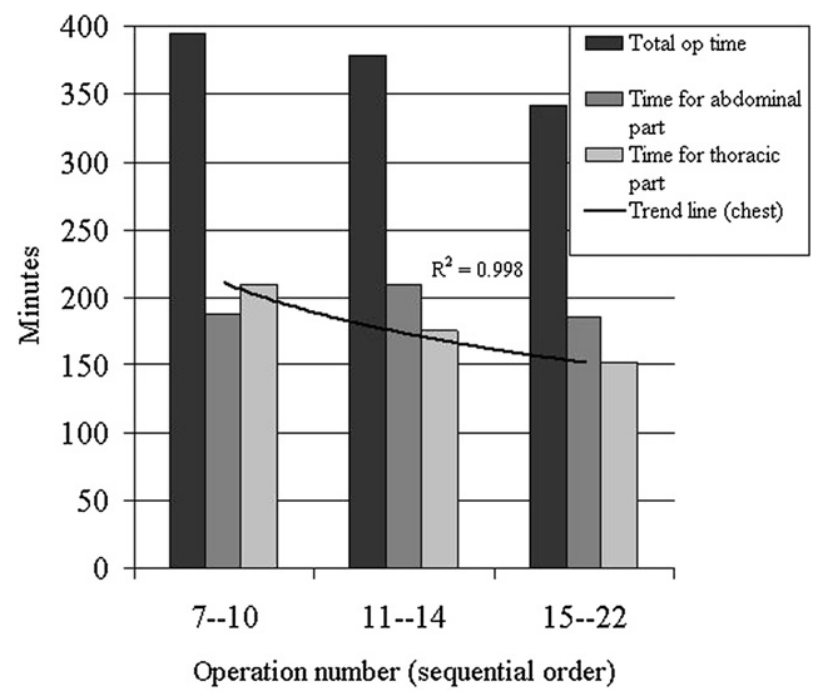

FIGURE 3. The median operative times for the last 16 patients (all of whom underwent a robotic hand-sewn, 2-layered, thoracic anastomosis). Logarithmic trend line for the thoracic aspect of the operation is shown $\left(R^{2}=0.998\right)$.

In this study, we depicted our preferred port placement and anastomotic technique. We honestly reported our poor results using a stapled posterior and hand-sewn anterior technique. Part of the improvement over time in this series may have been attributable to the learning curve-the patients who had a hand-sewn anastomosis were all treated after the patients who had a stapled anastomosis. We favor the 2-layered hand-sewn technique for the reasons described earlier.

In this study, we have shown excellent early results using this latter technique. We have also shown that a CPR-ILE-4 is safe. It offers an outstanding platform to perform an MIE and affords the surgeon the opportunity to perform a complete thoracic lymphadenectomy, as shown by the high number of median lymph nodes resected in this series. Additionally, it is one of the few MIS approaches that allows an adroit, completely hand-sewn 2-layered esophagogastric anastomosis in the chest. The disadvantage of this technique is the time required to create the anastomosis. Other disadvantages include those associated with robotics, such as cost, the learning curve for the surgeon and his or her team, and the limited robotic platform availability in many hospitals. Although we continue to reduce our operative times, our technique is still probably longer than an experienced team that prefers to staple.

Many lessons were learned. First, the delivery of the gastric conduit into the chest via robotic instruments alone can traumatize the stomach's staple line. We have seen serosal tears and 1 patient who had a gastric leak (but an intact anastomosis). The robotic instruments used to deliver the conduit into the chest may have injured the gastric staple line. The small robotic instruments currently available are not suited for this maneuver alone. After our first 4 CPR-ILE-4 procedures, we now have our bedside nurse assistant grab the tubularized gastric conduit using a Scanlan lung clamp. The purchase is across the entire gastric tube and the conduit is transported into the chest from the abdomen in this way.

The next important technical aspects of the operation include the type and length of sutures used and the types of robotic instruments used to sew the anastomosis. Early in our experience we chose 2 robotic needle graspers for both the right and left hand. We noted that when following a running suture with a left-handed needle driver the suture was often frayed or torn. We also noted that we could not reposition the needle on the needle driver to different angles. Thus we switched the instrument in our left hand (robotic arm 2) to a long-tipped robotic forceps. This change solved both of these problems. However, it limited sewing to 1 hand and eliminated 1 of the great advantages of the robot- the ease of sewing with both hands. Another move that speeds the operation is to use a robotic needle driver in robotic arm 1 that has small suture cutters in its base. This avoids having to continually place robotic scissors in and out of robotic arm 1. The length of the suture and type of needle used are also very important and are described in the Methods section. These details can save time and reduce frustration.

The strengths of this article revolve mainly around the current clinical demand for details on how to do a robotic Ivor Lewis esophagogastrectomy and the large number of specifics details provided in this manuscript. This is a detailed technical paper from the experience of 1 robotic surgeon and his team, and we hope that the information in this manuscript will help to reduce the learning curve of others and to avoid some of our many mistakes, pitfalls, and frustrations. The limitations of this article include the short follow-up, the fact the series is relatively small, that it is from 1 thoracic surgeon, and that it is from a single institution. The short follow-up makes survival and diseasefree data meaningless, but this was not the purpose of this report.

We have shown that a CPR-IVE using 4 robotic arms (CPR-IVE-4) is safe, feasible, and appears to be oncologically sound, given that it offers an R0 resection even for large T3 and T4 tumors after neoadjuvant chemoradiotherapy. Our current port placement, as shown in Figure 1, $A$ and $B$, is reproducible, uses all 4 robotic arms, and avoid conflicts between the arms. Mobilization of the esophagus from the aorta is safe and yields extremely low blood loss. In addition, a completely hand-sewn 2-layered anastomosis is feasible and early results are favorable. Many specific technical tricks can be used to reduce the operative times and frustration. Further studies and modification in operative techniques are needed. In addition, long-term follow-up data on survival and disease-free are also needed. 


\section{References}

1. Carr SR, Luketich JD. Minimally invasive esophagectomy. An update on the options available. Minerva Chir. 2008;63:481-95.

2. Sgourakis G, Gockel I, Radtke A, Musolt TJ, Timm S, Rink A, et al. Minimally invasive versus open esophagectomy: Meta Analysis of Outcomes. Dig Dis Sci. 2010;55:331-40.

3. Walther B, Johansson J, Johnsson F, Von Holstein CS, Zilling T. Cervical or thoracic anastomosis after esophageal resection and gastric tube reconstruction: a prospective randomized trial comparing sutured neck anastomosis with stapled intrathoracic anastomosis. Ann Surg. 2003;238:803-12.

4. Okuyama M, Motoyama S, Suzuki H, Saito R, Maruyama K, Ogawa J. Handsewn cervical anastomosis versus stapled intrathoracic anastomosis after esophagectomy for middle or lower thoracic esophageal cancer: a prospective randomized controlled study. Surg Today. 2007;37:947-52.

5. Levy RM, Luketich JD. Laparoscopic and thoracoscopic esophagectomy. Semin Thorac Cardiovasc Surg. 2010;22:256-8.

6. Levy RM, Wizorek J, Shende M, Luketich JD. Laparoscopic and thoracoscopic esophagectomy. Review. Adv Surg. 2010;44:101-16.

7. Orringer MB, Marshall B, Iannettoni MD. Transhiatal esophagectomy: clinical experience and refinements. Ann Surg. 1999;230:392-400.

8. Cerfolio RJ, Bryant AS, Canon CL, Dhawan R, Eloubeidi MA. Is botulinum toxin injection of the pylorus during Ivor Lewis esophagogastrectomy the optimal drainage strategy? J Thorac Cardiovasc Surg. 2009;137:565-72.

9. Bryant AS, Rudemiller K, Cerfolio RJ. The 30- versus 90-day operative mortality after pulmonary resection. Ann Thorac Surg. 2010;89:1717-22; discussion 1722-3.

10. Orringer MB, Marshall B, Iannettoni MD. Eliminating the cervical esophagogastric anastomotic leak with a side-to-side stapled anastomosis. $J$ Thorac Cardiovasc Surg. 2000;119:277-88.

11. Gorenstein LA, Bessler M, Sonett JR. Intrathoracic linear stapled esophagogastric anastomosis: an alternative to the end to end anastomosis. Ann Thorac Surg. 2011;91:314-6.

12. Kernstine KH, DeArmond DT, Kaimi M, Van Natta TL, Campos JH, Yoder MR, et al. The robotic, 2-stage, 3-field esophagolympadenectomy. J Thorac Cardiovasc Surg. 2004; 127:1847-9.

13. Bodner JC, Zitt M, Ott H, Wetscher GJ, Wykypie H, Lucciarini P, et al. Roboticassisted thoracoscopic surgery for benign and malignant esophageal tumors. Ann Thorac Surg. 2005;80:1202-6.

14. Boone J, Schipper MEI, Moojen WA, Rinkes IHMB, Cromheecke GJE, van Hillegersberg R. Robot-assisted thoracoscopic oesophagectomy for cancer. $\mathrm{Br}$ J Surg. 2009;96:878-86.

15. Puntambekar SP, Rayate N, Joshi S, Agarawal G. Robotic transthoracic esophagectomy in the prone position: experience with 32 patients with esophageal cancer. J Thorac Cardiovasc Surg. 2011;142:1283-4.

16. Weksler B, Sharma P, Moudgill N, Chojnacki KA, Rosato EL. Robot-assisted minimally invasive esophagectomy is equivalent to thoracoscopic minimally invasive esophagectomy. Dis Esoph. 2011 (in press). 